\title{
Magnetic Reconnection with Sweet-Parker Characteristics in Two-dimensional Laboratory Plasmas
}

Hantao Ji, Masaaki Yamada, Scott Hsu, Russell Kulsrud, Troy Carter, and Sorin Zaharia

Plasma Physics Laboratory, Princeton University, P. O. Box 451, Princeton, NJ 08543

\begin{abstract}
Magnetic reconnection has been experimentally studied in a well-controlled, two-dimensional laboratory magnetohydrodynamic plasma. The observations are found to be both qualitatively and quantitatively consistent with a generalized Sweet-Parker model which incorporates compressibility, downstream pressure, and the effective resistivity. The latter is significantly enhanced over its classical values in the collisionless limit. This generalized SweetParker model also applies to the case in which an unidirectional, sizable third magnetic component is present.
\end{abstract}

PACS numbers: 52.30.Jb, 96.60.Rd, 94.30.Lr

Typeset using REVTEX 


\section{INTRODUCTION}

Magnetic reconnection is the topological change of a magnetic configuration through breaking and rejoining of magnetic field lines. It plays a crucial role in determining the topology of magnetic fields in space and laboratory plasmas ${ }^{1-3}$. Although this is a localized process, it often causes fundamental changes in macroscopic configurations, such as in solar flares ${ }^{4}$, magnetospheric substorms ${ }^{4}$, and relaxation processes in laboratory plasmas ${ }^{5}$. Magnetic reconnection also provides the most plausible mechanism for releasing the energy stored in the magnetic field to plasma kinetic and thermal energies as observed in solar flares, auroral phenomena, and laboratory plasmas.

Magnetic reconnection was first suggested more than 50 years ago ${ }^{6}$ in order to explain activities associated with observed solar flares. Long and quiet periods (days to months) exist before a sudden (minutes to hours) explosion of a solar flare. Rapid changes in macroscopic structures associated with strong magnetic fields have been a mystery since they were first observed more than 40 years ago. Sweet $^{7}$ and Parker $^{8}$ separately proposed the first quantitative model of magnetic reconnection in two-dimensional geometry to solve this mystery. This model was rather revolutionary in the sense that it was shown for the first time how localized "reconnection" of field lines can cause the observed macroscopic changes.

Soon after it was proposed, however, it was realized that the Sweet-Parker model gives a characteristic time too slow to explain solar flares. A typical Alfvén time $\tau_{A}$ is on the order of $1 \mathrm{~s}$ while the resistive diffusion time $\tau_{R}$ is on the order of $10^{14} \mathrm{~s}$, resulting in a Lundquist number $S \equiv \tau_{R} / \tau_{A} \approx 10^{14}$. The Sweet-Parker model predicts a time-scale of $\sqrt{\tau_{A} \tau_{R}} \approx 10^{6}-10^{7} \mathrm{~s}$ (see the next Section) for macroscopic changes to take place, compared a typical time-scale of $10^{3}-10^{4} \mathrm{~s}$ of solar flares. Because of this discrepancy, the attention has shifted to Petschek's model ${ }^{9}$ and other models based on a much smaller diffusion region and standing wave structures $(\text { shocks })^{10,11}$. The much smaller size of the diffusion region allows a much faster reconnection rate which can be consistent with observations. The Petschek model has since been favored over the Sweet-Parker model - especially because of its faster 
predicted reconnection rates.

Despite the theoretical and computational progress made in past decades on magnetic reconnection, all these models have remained unchallenged by a decisive MHD plasma experiment in an appropriate geometry. Stenzel and Gekelman ${ }^{12}$ carried out a series of experiments in a linear device and in the electron magnetohydrodynamic (EMHD) regime where only electrons are magnetized while most space plasmas of interest are in the MHD regime where ions are also magnetized. Although detailed local fluctuations were measured in their experiments, quantitative tests of these leading two-dimensional (2D) MHD models were not possible. More recent experiments have focused on the effects of the third field component during reconnection from both global ${ }^{13,14}$ and local ${ }^{15}$ points of view. In this article, we report such quantitative tests ${ }^{16}$ of the Sweet-Parker model in the Magnetic Reconnection Experiment $(\mathrm{MRX})^{17}$.

The MRX device is the most recent device dedicated to investigate the fundamental physics of magnetic reconnection in MHD plasmas. In MRX, both the local and global physics issues and their interrelationship are being extensively studied. The initial geometry is made to be axisymmetric (and hence two-dimensional) although it can be made nonaxisymmetric to study three-dimensional (3D) characteristics of reconnection. These plasmas have a high conductivity $\left(S \sim 10^{3}\right)$ with the ion gyro-radius being much smaller than the plasma size, satisfying conditions for MHD approximations. The well-controlled environment in MRX permits formation of well-defined, two-dimensional current sheets in a stable manner, enabling quantitative comparisons with 2D MHD models through approximations based on space-averaged analysis. A significant finding is that the observed reconnection rate can be explained by a generalized Sweet-Parker model which includes compressibility, downstream pressure, and the effective resistivity. The latter is significantly enhanced over its classical values in the collisionless limit.

Arrangement for other sections is the following. In Sec. II, a brief derivation of the Sweet-Parker model is given. In Sec. III, experimental apparatus of MRX including major diagnostics are described. After the presentation of the main results in Sec. IV, implications 
of the results will be discussed in Sec. V, followed by conclusions in Sec. VI.

\section{THE SWEET-PARKER MODEL}

A key element of the Sweet-Parker model is the existence of a "diffusion region" - essentially a rectangular box where the magnetic field diffuses and reconnects, as illustrated in Fig. 1. The dimensions of such a "box" are of crucial importance since it essentially decides the rate of magnetic reconnection by balancing incoming and outgoing plasma and flux flow and thus the time scale for reconnection. The length of this box is of macroscopic scale but its width is determined by the local plasma resistivity which causes magnetic diffusion; i.e., faster reconnection occurs with larger resistivity. The Sweet-Parker model uses resistivities estimated by classical theories, such as the Spitzer resistivity ${ }^{18}$.

The motion of magnetic field lines in an MHD plasma with resistivity $\eta$ is described by

$$
\frac{\partial \boldsymbol{B}}{\partial t}=\nabla \times(\boldsymbol{V} \times \boldsymbol{B})+\frac{\eta}{\mu_{0}} \nabla^{2} \boldsymbol{B}
$$

where $\boldsymbol{V}$ is the flow velocity. The first term on the right hand side represents the effect of plasma convection whose time-scale is Alfvén time $\tau_{A}=L / V_{A}$, where $L$ is the plasma size (or the length of diffusion region in the case of magnetic reconnection) and $V_{A} \equiv B / \sqrt{\mu_{0} \rho}$ ( $\rho=$ mass density) is the Alfvén speed. The second term describes field line diffusion whose time-scale is the diffusion time $\tau_{R}=\mu_{0} L^{2} / \eta$. The relative importance of magnetic diffusion to plasma convection is given by the Lundquist number $S$ defined by $\tau_{R} / \tau_{A}=\mu_{0} L V_{A} / \eta$. For typical MHD plasmas such as solar flares ${ }^{4}, S>10^{10}$; for tokamaks, $S>10^{7}$; and for MRX plasmas, $S \lesssim 10^{3}$.

Another important equation governing reconnection is the continuity equation,

$$
\frac{\partial n}{\partial t}+\boldsymbol{\nabla} \cdot(n \boldsymbol{V}) \approx \frac{\partial \bar{n}}{\partial t}-\frac{\bar{n} V_{R}}{\delta}+\frac{\bar{n} V_{Z}}{L}=0
$$

where $\delta$ is the thickness (or width of the diffusion region) of the current sheet as shown in Fig. 1 and $\bar{n}$ is the averaged density in the diffusion region. Figure 1 also indicates $V_{R}\left(V_{Z}\right)$ 
as plasma flow speed across (along) the diffusion region. These flows are in the radial $(R)$ and axial $(Z)$ direction, respectively, reflecting the geometry in MRX (see the next Section).

The last relevant equation is the equation of motion,

$$
\rho\left(\frac{\partial}{\partial t}+\boldsymbol{V} \cdot \boldsymbol{\nabla}\right) \boldsymbol{V}=-\boldsymbol{\nabla} p+\boldsymbol{j} \times \boldsymbol{B} .
$$

Integration of this equation, (i.e., the $R$ component across the current sheet and the $Z$ component along the current sheet) gives

$$
\begin{aligned}
& \int_{0}^{\delta} \rho \frac{\partial V_{R}}{\partial t} \mathrm{~d} R+p_{\text {up }}+\frac{1}{2} \rho V_{R}^{2}+\frac{B_{Z}^{2}}{2 \mu_{0}}-\frac{1}{\mu_{0}} \int_{0}^{\delta} B_{Z} \frac{\partial B_{R}}{\partial Z} \mathrm{~d} R=p_{0} \\
& =\int_{0}^{L} \rho \frac{\partial V_{Z}}{\partial t} \mathrm{~d} Z+p_{\text {down }}+\frac{1}{2} \rho V_{Z}^{2}+\frac{B_{R}^{2}}{2 \mu_{0}}-\frac{1}{\mu_{0}} \int_{0}^{L} B_{R} \frac{\partial B_{Z}}{\partial R} \mathrm{~d} Z,
\end{aligned}
$$

where $p_{0}, p_{\text {up }}$ and $p_{\text {down }}$ are plasma pressures at the center and in the upstream and downstream regions, respectively. The last terms on both sides represent the magnetic tension forces.

The original Sweet-Parker model ${ }^{7,8}$ assumes steady state reconnection $(\partial \boldsymbol{B} / \partial t=0$, $\partial \boldsymbol{V} / \partial t=0)$ in an incompressible plasma $(\boldsymbol{\nabla} \cdot \boldsymbol{V} \propto \partial \bar{n} / \partial t=0)$ with uniform pressure outside the diffusion region $\left(p_{\text {up }}=p_{\text {down }}\right)$ and with negligible $\rho V_{R}^{2} / 2, B_{R}^{2} / 2 \mu_{0}$, and tension forces. Then Eqs. (1), (2) and (4) can be reduced to $V_{R}=\eta / \mu_{0} \delta, V_{R}=(\delta / L) V_{Z}$ and $V_{Z}=V_{A}$, resulting in a simple expression for the reconnection rate as measured by the Alfvén Mach number, $M_{A} \equiv V_{R} / V_{A}=\sqrt{\eta / \mu_{0} L V_{A}}=1 / \sqrt{S}$. Therefore, a characteristic time given by the model is $\tau_{S P}=L / V_{R}=L / V_{A} \sqrt{S}=\sqrt{\tau_{A} \tau_{R}}$.

\section{EXPERIMENTAL APPARATUS AND DIAGNOSTICS}

Since a detailed description of the MRX device has been given in a previous paper ${ }^{17}$, only major relevant parts are briefly mentioned here. When a plasma is inductively formed by two internal coils (called the flux cores) in a quadrupole field configuration, the magnetic field domain can be divided into three sections: two private sections surrounding each flux core and one public section surrounding both flux cores [see Fig. 2(a)]. When poloidal flux 
in the private sections is reduced the poloidal flux is "pulled" back from the public section to the private sections resulting in magnetic reconnection as shown in Fig. 2(b). Toroidally symmetric shape of the flux cores ensures global 2D geometry for magnetic reconnection.

The low temperature $(<50 \mathrm{eV})$ and short-pulsed $(<1 \mathrm{~ms})$ MRX plasmas have the advantage that internal probes can be used routinely. Triple Langmuir probes are used to measure electron density $\left(n_{e}\right)$ and temperature $\left(T_{e}\right)$ simultaneously. The plasma density measurement has been calibrated by a laser interferometer which measures the line-integrated density. All three components of $\boldsymbol{B}$ are measured during the reconnection process by a 90 channel 2D pick-up coil array with $4 \mathrm{~cm}$ resolution. The poloidal flux function can be obtained by integration of $B_{Z}$ over the radius, $\Psi(R, Z)=2 \pi \int_{0}^{R} B_{Z}(R, Z) R d R$. A finer 1D pick-up probe array with $0.5 \mathrm{~cm}$ resolution is used to measure the $B_{Z}$ profile across the current sheet ${ }^{15}$. Local flow velocity can be determined either by a Mach probe or time evolution of $\Psi(R, Z)$, i.e., $V_{X}=-(\partial \Psi / \partial t) /(\partial \Psi / \partial X)(X=R$ in the upstream region and $X=Z$ in the downstream region). The latter method is valid when the resistive effects are negligible, a condition satisfied outside the diffusion region. Results from both methods are in good agreement, and the latter has been used routinely because of its convenience. Probe perturbation of the plasma is estimated quantitatively and observed to be less than $5 \%{ }^{17}$. Typical plasma parameters are as follows: $B<0.5 \mathrm{kG}, T_{e}=5-20 \mathrm{eV}$, and $n_{e}=0.2-1.5 \times 10^{20} \mathrm{~m}^{-3}$.

\section{EXPERIMENTAL TESTS OF THE SWEET-PARKER MODEL}

Because of the incompressibility assumption, the effects of the third component (perpendicular to the page in Fig. 1) of the reconnecting magnetic field vector do not enter explicitly in the Sweet-Parker model. However, the third component has been identified

as an important factor in deciding reconnection rate ${ }^{13,15}$. When the third component (the toroidal field $B_{T}$ in $\left.\mathrm{MRX}\right)$ is negligibly small compared to the reconnecting field $\left(B_{Z}\right)$, i.e. the null-helicity case, the reconnection proceeds faster, while in the case with a sizable $B_{T}$, i.e. the co-helicity case, a slower reconnection rate has been observed. In the following sec- 
tions, the results from these cases are presented separately in comparison with predictions from the Sweet-Parker model.

\section{A. The Null-helicity Case}

An example of driven magnetic reconnection in MRX is displayed in Fig. 3(a) and (b), where both the measured magnetic field vector $\boldsymbol{B}$ and contours of the poloidal flux $\Psi$ in a single discharge are plotted in an $R-Z$ plane. Qualitatively, this double-Y-shaped diffusion region is consistent with the Sweet-Parker assumption on the existence of a rectangular diffusion region. Quantitative tests of the Sweet-Parker model, however, requires adequate measurements of all the basic plasma parameters.

In order to accurately determine current sheet thickness $\delta$ and peak current density, the reconnecting $B_{Z}$ profiles across the current sheet are measured at $Z=0$ by the fine 1D probe array. The measured $B_{Z}$ profiles are well fit into the Harris-type function ${ }^{19}$, $\tanh \left[\left(R-R_{0}\right) / \delta\right]$, as shown in Fig. 3(c) and (d). A shot-averaged time evolution of several key plasma parameters for driven reconnection is shown in Fig. 4. The current density peaks at $t=290 \mu \mathrm{s}$, when $\delta$ is minimized and reconnection speed $V_{R}$ reaches its steady state of about $3 \mathrm{~km} / \mathrm{s}$. The $n_{e}$ measured at the center of the current sheet keeps increasing until a later time, while $T_{e}$ at the same location remains almost constant at $10-15 \mathrm{eV}$. In general, the Lundquist number $S$ is calculated from the measured $T_{e}$ based on the Spitzer resistivity ${ }^{18}$ (parallel resistivity, $\eta_{\|}$). This is true for the case of co-helicity reconnection where the current flows along a sizable third component in the diffusion region. However, perpendicular resistivity $\eta_{\perp}\left(=2 \eta_{\|}\right)$should be used in the case of null-helicity reconnection since the current flows essentially perpendicular to the field. A more detailed calculation which incorporates profile effects of density and temperature gives a nearly identical expression for resistivity ${ }^{20}$.

A series of experiments has been performed in which $B_{Z}$ is varied from $200 \mathrm{G}$ to 420 $\mathrm{G}$ while other conditions are kept constant, including the fill pressure $p_{\text {fill }}$ (6 mTorr). It is

observed that the reconnection rate decreases as $B_{Z}$ increases. A straightforward test of 
the Sweet-Parker model is shown in Fig. 5 where the reconnection rate is plotted against $1 / \sqrt{S}$. Clearly, the observation does not agree with the Sweet-Parker prediction (dotted line). While the reconnection rate changes by a factor of $3,1 / \sqrt{S}$ changes only by a factor of 1.5. Causes of these discrepancies can be found by systematically examining the validity of each assumption made by Sweet and Parker in Eqs. (1), (2), and (4).

The first equation to be evaluated is Ohm's law in the toroidal direction, $E_{T}+V_{R} \times B_{Z}=$ $\eta_{\perp} j_{T}$, which has been used to derive Eq. (1). All three terms are measured across the current sheet. As shown in the inset of Fig. $6, E_{T}(=-\dot{\Psi} / 2 \pi R)$ balances with $V_{R} \times B_{Z}$ outside the diffusion region and $\eta_{\perp} j_{T}$ inside the diffusion region. In this example, the measured effective resistivity $\left(\eta_{\perp}^{*}=E_{T} / j_{T}\right)$ is about twice its classical value. It is found that the enhancement of resistivity is a strong function of collisionality (characterized by the dimensionless parameter $\lambda_{\operatorname{mfp}} / \delta$ and dominated by changes in density), as shown in Fig. 6 . A significant enhancement $(\sim 10)$ of the resistivity is observed in the collisionless regime $\left(\lambda_{\operatorname{mfp}} \gg \delta\right)$.

We note that electron-neutral collisions are estimated to be negligible compared to Coulomb collisions in the present experimental regimes. For $T_{e}=10-15 \mathrm{eV}$, the estimated cross-section of electron-neutral collision is $7 \times 10^{-17} \mathrm{~cm}^{2}$ including all possible processes ${ }^{21}$. With the maximum possible neutral density of $4 \times 10^{14}$ atom $/ \mathrm{cm}^{3}$ from $p_{\text {fill }}=6$ mTorr, the mean free path for electron-neutral collisions is $\geq 35 \mathrm{~cm}$, much longer than $2-14 \mathrm{~cm}$ of mean free path due to Coulomb collisions.

The incompressibility assumption does not hold since the $\boldsymbol{\nabla} \cdot \boldsymbol{V}$ (or $\partial \bar{n} / \partial t$ ) term is not negligible compared to the other terms in the continuity equation [Eq.(2)] as seen in Fig. 4, which shows increasing density in the current sheet during reconnection. Retaining this term in the continuity equation leads to an increased inflow,

$$
V_{R}=\frac{\delta}{L}\left(V_{Z}+\frac{L}{n} \frac{\partial n}{\partial t}\right)
$$

due to an accumulation of density at the center. Quantitative importance of compressibility is shown in Fig. 7, where the ratio of the second term to the first term of the above equation is plotted against $M_{A}=V_{R} / V_{A}$. The accumulation of density can result in an increase 
in $V_{R}$ by as large as $40 \%$ especially in the cases of larger $B_{Z}$ or narrower current sheet. Compressibility, which allows local density build-up, explains why higher central density is observed in discharges with higher field even though the initial density is the same. We note that the particle source term in the diffusion region is negligible in the present experimental regimes. [It appears that in discharges with high $p_{\text {fill }}(\gtrsim 10 \mathrm{mTorr})$, the continuity equation cannot be satisfied without an additional source term.]

Examination of each term in the momentum equation [Eq. (4)] reveals that all assumptions made in the Sweet-Parker model hold approximately true, except that $p_{\text {down }} \gg p_{\text {up }}$ (dominated by differences in density), as measured by spatial scans of a Langmuir probe. Figure 8 shows time evolution of densities measured at center $(Z=0, R=37.5 \mathrm{~cm})$, upstream $(Z=0, R=30 \mathrm{~cm}$ and $44 \mathrm{~cm})$, and down-stream $(Z=-10 \mathrm{~cm}, R=37.5 \mathrm{~cm})$ regions. The down-stream density increases in time and becomes comparable to the central density later into reconnection while the up-stream density stays low and constant. The measured electron temperatures are roughly flat over space. Therefore, the momentum equation is modified to

$$
V_{Z}^{2}=V_{A}^{2}(1+\kappa)-2 \frac{p_{\text {down }}-p_{\text {up }}}{\rho}
$$

where $\kappa \equiv\left(2 / B_{Z}^{2}\right) \int_{0}^{L} B_{R}\left(\partial B_{Z} / \partial R\right) \mathrm{d} Z$ representing the relative importance of the downstream tension force, which is omitted in the Sweet-Parker model. Calculated from the measured profiles of $B_{R}$ and $B_{Z}$, values of $\kappa$ range from 0.2 to 0.3 , leading a slight $(\sim 0.1)$ increase in the outflow. However, the higher down-stream pressure $p_{\text {down }} \sim p_{0} \gg p_{\text {up }}$ substantially reduces the outflow, as also shown in in Fig. 5. As $S$ increases, the reconnection rate decreases and the outflow slows to as low as 10-20\% of $V_{A}$.

By incorporating the effective resistivity, compressibility, and finite down-stream pressure, the Sweet-Parker model can be modified to define an effective Lundquist number $S_{\text {eff }}$ as

$$
S_{\text {eff }}=\frac{\mu_{0} L V_{A}}{\eta^{*}} \cdot \frac{1}{1+L \dot{n} / n V_{Z}} \cdot \frac{V_{Z}}{V_{A}}
$$


so that $M_{A}=1 / \sqrt{S_{\text {eff. }}}$. The observed reconnection rate is plotted against $1 / \sqrt{S_{\text {eff }}}$ in Fig. 9 . As expected, they are in good agreement. Indeed, the process of magnetic reconnection in MRX can be explained quantitatively by a generalized version of the Sweet-Parker model.

\section{B. The Co-helicity Case}

In general, the third vector component may not be negligibly small compared with the reconnecting components during magnetic reconnection happening in nature. Without the third component (the null-helicity case), the reconnecting field lines are exactly anti-parallel, while in the presence of an uni-directional, sizable third component (the co-helicity case), the field lines reconnect obliquely. In the classical Sweet-Parker model described in Sec. II, the effects of the third component of $\boldsymbol{B}$ do not enter because of the incompressible assumption. Since the generalized Sweet-Parker model is applicable to the null-helicity case in MRX, an interesting question is whether or not the same model can also explain the observed reconnection rate in the co-helicity case.

Effects of the third component have been extensively studied in $\mathrm{MRX}^{15}$. Two major differences have been observed during "pull" reconnection experiments in MRX: (1) a thin double-Y-shaped diffusion region forms in the null-helicity case while an O-shaped diffusion region develops in the co-helicity case; (2) the reconnection proceeds much slower in the co-helicity than in the null-helicity even all other conditions are held constant.

In order to apply the Sweet-Parker model, a diffusion region with the shape of a rectangular box needs to be well-defined. At the first glance, unlike the null-helicity case, it would appear that a diffusion region with an O-point is inconsistent with the Sweet-Parker assumption of a rectangular box. However, a careful examination of the current density profiles shown in Fig. 10(c) reveals that the thickness of current sheet is indeed well-defined and it is almost independent of $Z$. The O-shaped fine structure resides well within the current sheet and it is not important in defining a rectangular diffusion region. The profiles of $B_{Z}, B_{T}$, and $j_{T}$ at $Z=0$ are shown in Fig. 10(a) and (b). Again, $\delta$ is determined by 
fitting $j_{T}$ into the Harris-type current sheet ${ }^{19}, \operatorname{sech}^{2}\left[\left(R-R_{0}\right) / \delta\right]$.

As in the null-helicity case, all three modifications to the original Sweet-Parker model have been examined for the co-helicity case. It is noted that $\eta_{\|}$should be used here to calculate the classical resistivity. It is found that the resistivity enhancement ranges $2-4$ for the cases examined so far at a relatively low field $\left(B_{Z} \approx 200 \mathrm{G}\right)$ while the collisionality parameter $\lambda_{\mathrm{mfp}} / \delta=0.5-2$. Density accumulation in the diffusion region is negligible in this case, as expected from the fact that the existence of a sizable $B_{T}$ makes plasma less compressible. The effect of higher down-stream pressure than up-stream is more predominant, resulting in an outflow as low as $\sim 5 \%$ of the Alfvén speed.

The generalized Sweet-Parker model applies also to the co-helicity cases, as shown in Fig. 11, where the observed reconnection rates in both null- and co-helicity cases are plotted against $1 / \sqrt{S_{\text {eff }}}$, spanning over a decade in magnitude. The reconnection rate in the cohelicity case is slower than the null-helicity case due to a combined effect of lower anomalous resistivity, lower compressibility, and higher down-stream pressure.

\section{DISCUSSIONS}

Qualitatively, magnetic reconnection observed in MRX is in good accord with the SweetParker model whose essence resides in the existence of a rectangular diffusion region. Such a rectangular box is well-defined experimentally in a quasi-steady state manner regardless of the existence of the third component. The global two-dimensional nature of magnetic reconnection is ensured by the axisymmetric boundary conditions although the fine-scale dynamics within the diffusion region, such as microinstabilities, must be fully three-dimensional.

Quantitatively, the observed reconnection rates can also be explained by the SweetParker model but only after it is generalized to incorporate three effects: effective resistivity, compressibility and finite down-stream pressure. One relevant question might be whether Petschek-type models can explain the same observations. Direct comparisons, however, are not possible since these shock-based models do not predict definite reconnection rates, only 
their maxima ${ }^{2}$. Shock structures, a characteristic feature of Petschek-type models, would appear as multiple jumps in $B_{Z}(R)$ profiles in the downstream region. However, these jumps have not been observed yet within the sensitivity limits of the measurements. We note that the present work neither verifies nor disproves the Petschek-type model. Further experimental investigations in MRX include searching for shock structures in larger $S$ regimes or under more powerful driving forces through external coils.

All three effects incorporated in the generalized Sweet-Parker model can be important also during magnetic reconnection happening in nature or other laboratory plasmas. Effects of the compressibility must be transient (as seen in MRX) by nature since the density accumulation cannot be sustained indefinitely. However, occurrences of magnetic reconnection in nature do not have to be steady state. They can be impulsive locally while global structures are maintained in a quasi (slowly evolving) steady state, as supported by a recent computer simulation using compressible MHD equations ${ }^{22}$.

The effect of downstream pressure is easy to understand. As observed in MRX, higher plasma pressure in the downstream region slows the outflow, thus reducing the reconnection rate. One can envision another case in which lower pressure in the downstream region can result in super-Alfvénic outflow, leading to an increase in the reconnection rate, a situation which can exist in solar flares and other cosmic environments.

Effects of finite viscosity have been also omitted during the analysis of the momentum equation in the Sweet-Parker model. However, this assumption cannot be justified when large velocity gradients exist in the down-stream region. In this case, a viscous term $\rho \nu_{\perp} \boldsymbol{\nabla}^{2} \boldsymbol{V}$ should be added to the right hand side of Eq.(3). Following W. Park ${ }^{23}$, a crude estimate of the viscous effects can be formulated to modify Eq.(6) to

$$
V_{Z}^{2}\left[1+\left(1+\frac{L}{n} \frac{\dot{n}}{V_{Z}}\right) \frac{\nu_{\perp} \mu_{0}}{\eta_{\perp}^{*}}\right]=V_{A}^{2}(1+\kappa)-2 \frac{p_{\text {down }}-p_{\text {up }}}{\rho},
$$

where the outflow could be also slowed by finite $\nu_{\perp}$. By using the classical values ${ }^{24}, \nu_{\perp} \mu_{0} / \eta_{\perp}$ can be estimated to

$$
\frac{\nu_{\perp} \mu_{0}}{\eta_{\perp}}=\frac{1}{4} \sqrt{\frac{m_{i}}{m_{e}}}\left(\frac{T_{e}}{T_{i}}\right)^{3 / 2} \beta_{i} \sim 1-2
$$


where $\beta_{i}=n T_{i} /\left(B^{2} / 2 \mu_{0}\right)$ is the ion beta. Therefore, the viscous effects are small if $\eta_{\perp}^{*} / \eta_{\perp} \gg$ 1. However, in order to quantify the $\boldsymbol{\nabla}^{2} \boldsymbol{V}$ term experimentally, measurements of detailed 2D velocity profiles are required.

A legitimate question regarding the relevance of laboratory plasma experiments to the space plasmas arises from the achievable low Lundquist numbers $\left(\sim 10^{3}\right)$ in laboratory. However, the observation of resistivity enhancement indicates that, just as in the space plasmas, the collisionless effects (within framework of either two-fluid treatments or full kinetic treatments of the diffusion region) dominate over the simple resistive MHD effects (Coulomb collisions) in MRX. Indeed, it is found that the current sheet thickness $\delta$ is determined not by Spitzer resistivity but by the ion skin depth $c / \omega_{p i}$, or equivalently the ion gyro-radius $\rho_{i}$ due to the force balance, $\beta^{*} \equiv\left(p_{0}-p_{\text {up }}\right) /\left(B_{Z}^{2} / 2 \mu_{0}\right)=1$. By using this force balance, the result $\delta \simeq \rho_{i}$ can be translated into a constant drift parameter, $v_{\mathrm{d}} / v_{\text {the }}$ $\left(v_{\mathrm{d}} / v_{\text {thi }}\right)$, where $v_{\mathrm{d}} \equiv j_{T} /$ en and $v_{\text {the }}\left(v_{\text {thi }}\right)=$ electron (ion) thermal velocity. In Fig. 12, the drift parameters are shown as functions of plasma density for the null-helicity case in MRX. Both drift parameters are relatively a constant, i.e., $v_{\mathrm{d}} / v_{\text {the }} \simeq 0.1$ and $v_{\mathrm{d}} / v_{\text {thi }} \simeq 3-4$, independent of density. This suggests that instabilities ${ }^{25}$ driven by relative drift between ions and electrons provide a mechanism to limit current density, thus controlling the reconnection rate. In the co-helicity case, a higher drift parameter $v_{\mathrm{d}} / v_{\text {the }} \sim 0.2$ is observed. However, we have to emphasize that the mechanism to limit current density should be different from the null-helicity case to the co-helicity case since the current essentially flows in the perpendicular direction in one case while parallel in the other. Finally, we note that these collisionless effects must enter the Ohm's law through fluctuations since all non-fluctuating terms (except $E_{T}$ and $\left.\eta j_{T}\right)$ in the generalized Ohm's law including the Hall and electron pressure terms are estimated to be negligible. Both theoretical and experimental undertaking invoking stability analysis and high frequency fluctuation measurements is underway in MRX in an effort to understand the mechanism of the resistivity enhancement. 


\section{CONCLUSIONS}

The well-controlled boundary conditions in MRX permit formation and maintenance of a stable, well-defined, two-dimensional current sheet (or diffusion region) during magnetic reconnection in MHD plasmas. In both qualitative and quantitative sense, the observed magnetic reconnection is consistent with a generalized Sweet-Parker model, independent of the presence of an unidirectional, sizable third magnetic component. The generalizations of the model include finite compressibility, higher down-stream pressure than up-stream, and the effective resistivity. The latter is significantly enhanced over its classical values in the collisionless limit. A significant implication of this result is that the Sweet-Parker model with generalizations is indeed valid in certain cases. By adopting the concept of anomalous resistivity, the Sweet-Parker model can predict a time-scale much closer to the one of the solar flares ${ }^{26}$, although we always have to keep in mind that the solar flares are much more complicated than just in a two-dimensional space. Nonetheless, laboratory experiments provide unique opportunities to challenge these theories in a well-controlled environment.

\section{ACKNOWLEDGMENTS}

The authors are grateful to D. Cylinder for his technical support. This work was jointly supported by the National Aeronautics and Space Administration, the National Science Foundation, the Office of Naval Research, and the Department of Energy. 


\section{REFERENCES}

${ }^{1}$ E. N. Parker, in Cosmical Magnetic Fields (Clarendon Press, Oxford, 1979).

${ }^{2}$ V. M. Vasyliunas, Rev. Geophys. Space Phys. 13, 303 (1975).

${ }^{3}$ D. Biskamp, Physics Report 237, 179 (1993).

${ }^{4}$ See, e.g., Introduction to Space Physics (Ed. by M. G. Kivelson and C. T. Russell, Cambridge University Press, 1995) and references therein.

${ }^{5}$ See, e.g., J. B. Taylor, Rev. Mod. Phys. 58, 741(1986).

${ }^{6}$ R. G. Giovanelli, Nature 158, 81 (1946).

${ }^{7}$ P. A. Sweet, in Electromagnetic Phenomena in Cosmical Physics, ed. B. Lehnert (Cambridge University Press, New York, 1958), pp.123.

${ }^{8}$ E. N. Parker, J. Geophys. Res. 62, 509 (1957).

${ }^{9}$ H. E. Petschek, NASA Spec. Pub. SP-50, 425 (1964).

${ }^{10}$ B. U. Ö. Sonnerup, J. Plasma Phys. 4, 161 (1970).

${ }^{11}$ E. R. Priest and T. G. Forbes, J. Geophys. Res. 91, 5579 (1986).

12 R. L. Stenzel and W. Gekelman, J. Geophys. Res. 86, 649 (1981).

${ }^{13}$ M. Yamada, Y. Ono, A. Hayakawa, M. Katsurai, F. W. Perkins, Phys. Rev. Lett. 65, 721 (1990).

${ }^{14}$ Y. Ono, M. Yamada, T. Akao, T. Tajima, R. Matsumoto, Phys. Rev. Lett. 76, 3328 (1996).

${ }^{15}$ M. Yamada, H. Ji, S. Hsu, T. Carter, R. Kulsrud, Y. Ono, and F. Perkins, Phys. Rev. Lett. 78, 3117 (1997).

${ }^{16}$ H. Ji, M. Yamada, S. Hsu, R. Kulsrud, Phys. Rev. Lett. 80, 3256 (1998). 
${ }^{17}$ M. Yamada, H. Ji, S. Hsu, T. Carter, R. Kulsrud, N. Bretz, F. Jones, Y. Ono, F. Perkins, Phys. Plasmas 4, 1936 (1997).

${ }^{18}$ L. Spitzer, Jr., Physics of Fully Ionized Gases (2nd Revised Edition), (Interscience Publishers, New York, 1962), p.28.

${ }^{19}$ E. G. Harris, Nuovo Cimento 23, 115 (1962).

${ }^{20}$ R. M. Kulsrud, Bull. Am. Phys. Soc. 42, 2028(1997).

${ }^{21}$ R. K. Janev, in Elementary Processes in Hydrogen-Helium Plasmas (Springer-Verlag, 1987).

${ }^{22}$ H. Kitabata, T. Hayashi, T. Sato, J. Phys. Soc. Jap. 65, 3208 (1996).

${ }^{23}$ W. Park, D. A. Monticello, and R. B. White, Phys. Fluids 27, 137 (1984).

${ }^{24}$ S. I. Braginskii, in Rev. of Plasma Physics 1 (Consultants Bureau, New York, 1966), p.218.

${ }^{25}$ See, e.g., A. T. Y. Lui, C.-L. Chang, A. Mankofsky, H.-K. Wong, and D. Winske, J. Geophys. Res. 96, 11389 (1991).

${ }^{26}$ R. M. Kulsrud, Phys. Plasmas 5, 1599 (1998). 


\section{FIGURES}

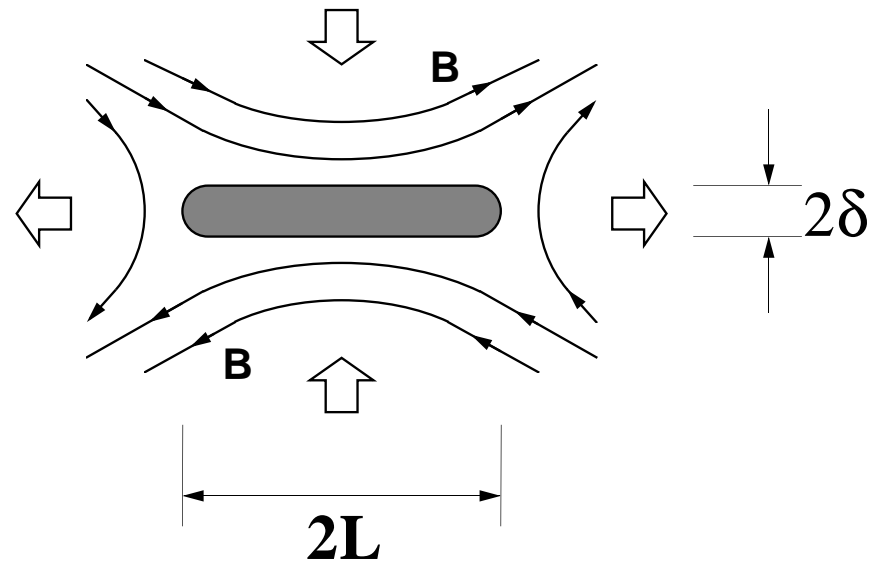

FIG. 1. An illustration of the Sweet-Parker model.

(a) Before reconnection

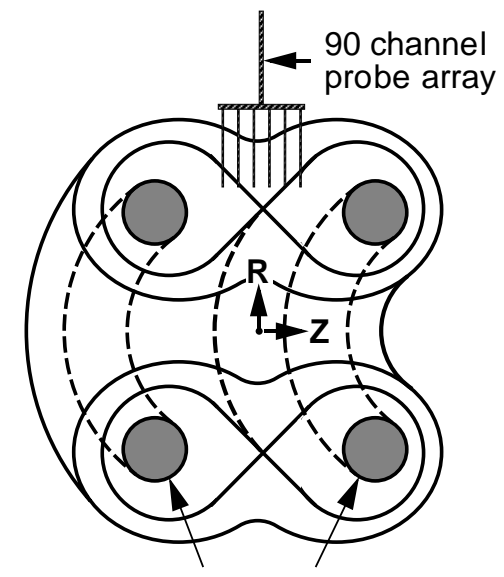

Flux core

(b) "Pull" reconnection

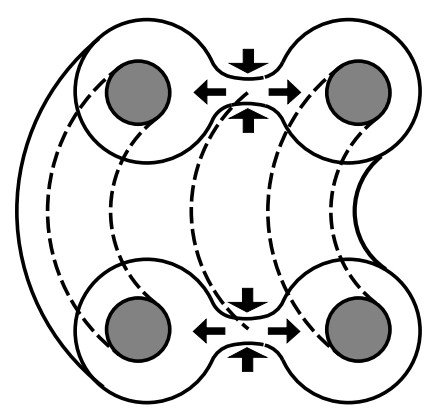

FIG. 2. An illustration of the MRX geometry for magnetic reconnection: (a) a quadrupole configuration formed by two flux cores, providing one public and two private regions, (b) a two-dimensional magnetic reconnection induced by pulling flux from the public back to the private regions. 

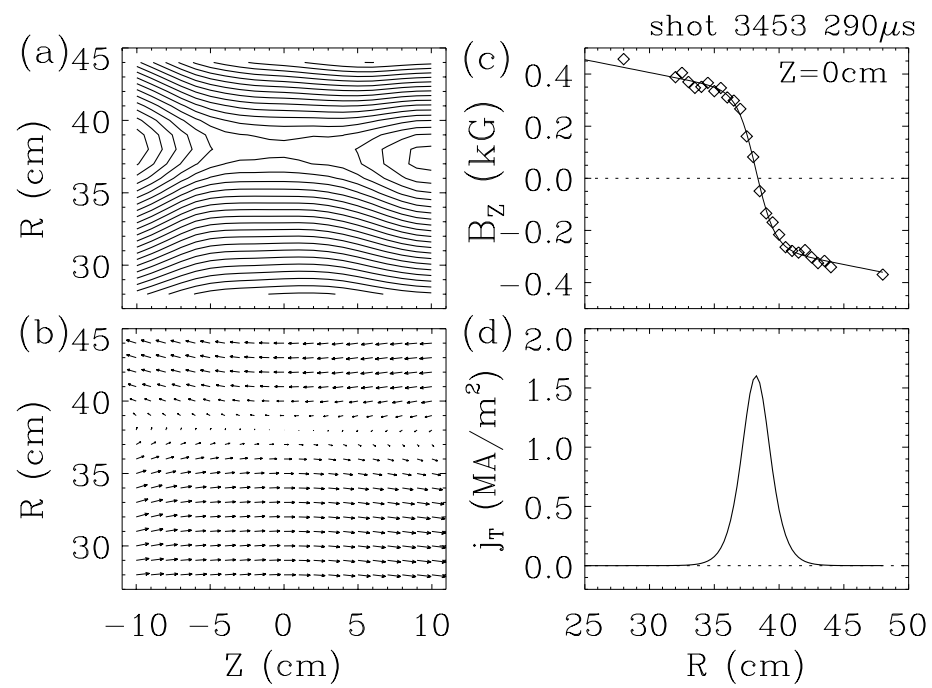

FIG. 3. An example of driven magnetic reconnection measured in a single shot by a $2 \mathrm{D}$ probe array: (a) vector plot of poloidal field, (b) poloidal flux contours, (c) measured radial profile of $B_{Z}$ by a fine probe array and fitted curve to $\tanh \left[\left(R-R_{0}\right) / \delta\right],(\mathrm{d})$ deduced current density profile. Toroidal field (the third component) is negligibly small.

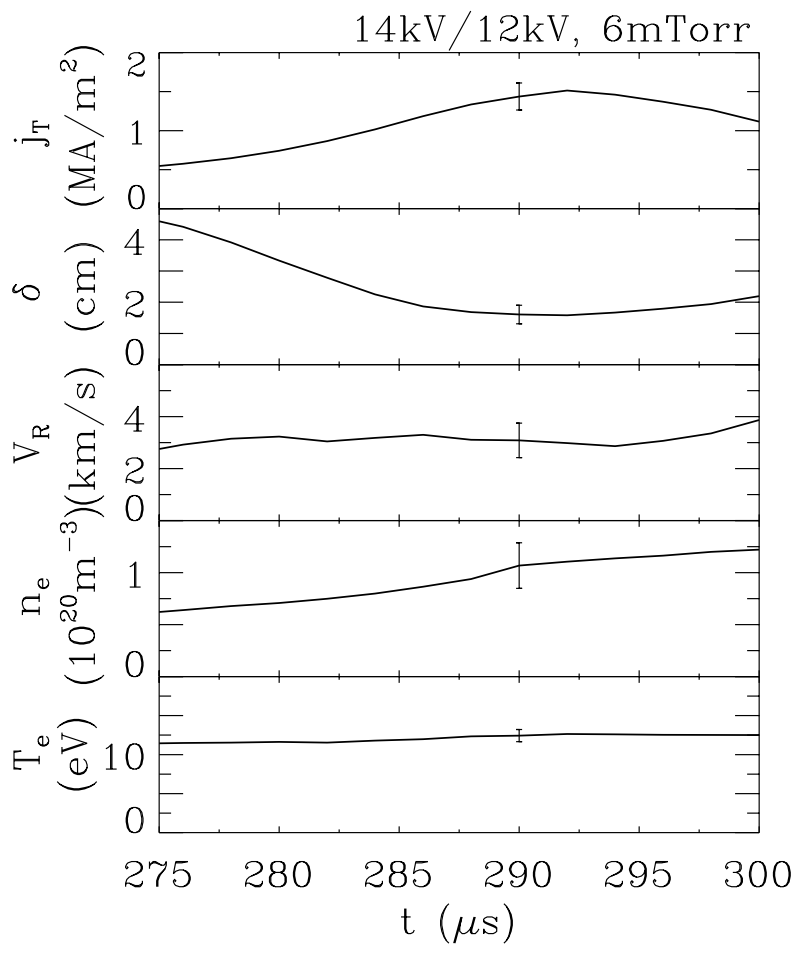

FIG. 4. A shot-averaged time evolution of driven magnetic reconnection. From top: peak current density, current sheet thickness, inflow speed at $R=30 \mathrm{~cm}$ from flux contour movement, electron density at center, electron temperature at center. 


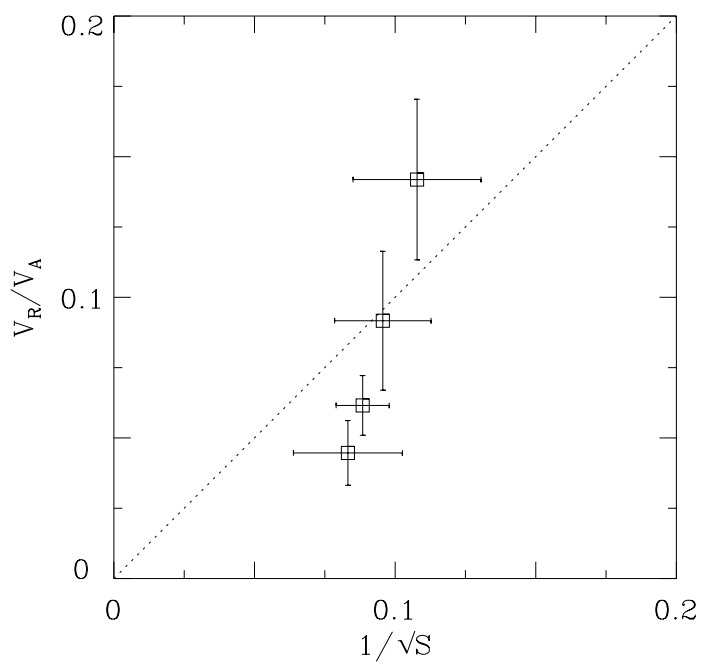

FIG. 5. Experimental test of the Sweet-Parker model (dotted lines): reconnection rate vs. $1 / \sqrt{S}$.

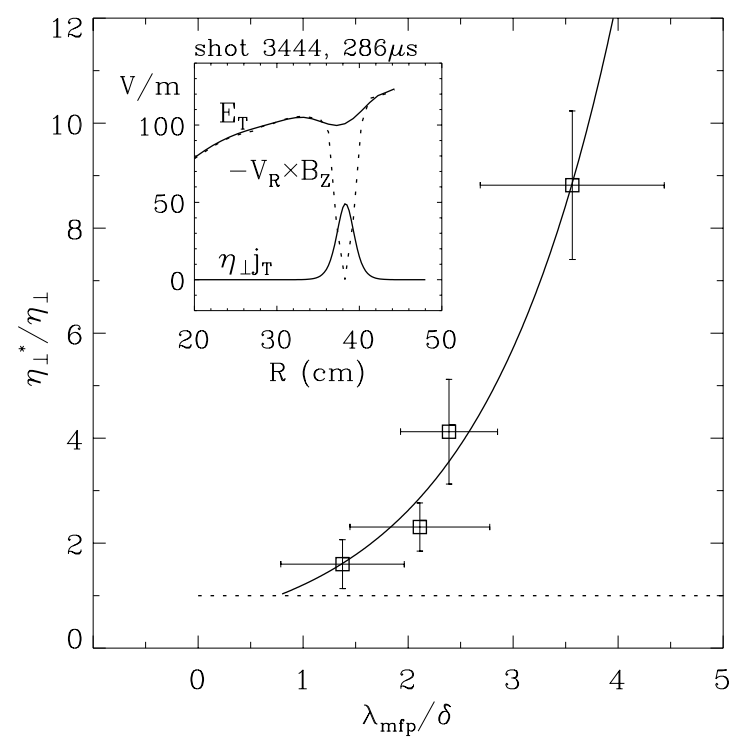

FIG. 6. Resistivity enhancement as a function of collisionality characterized by the ratio of electron mean free path (calculated from $n_{e}$ and $T_{e}$ ) to current sheet thickness. An example of all three terms of Ohm's law across the current sheet is shown in the inset where $E_{T}=-\dot{\Psi} / 2 \pi R$ and $V_{R}$ is from flux contour movement. 


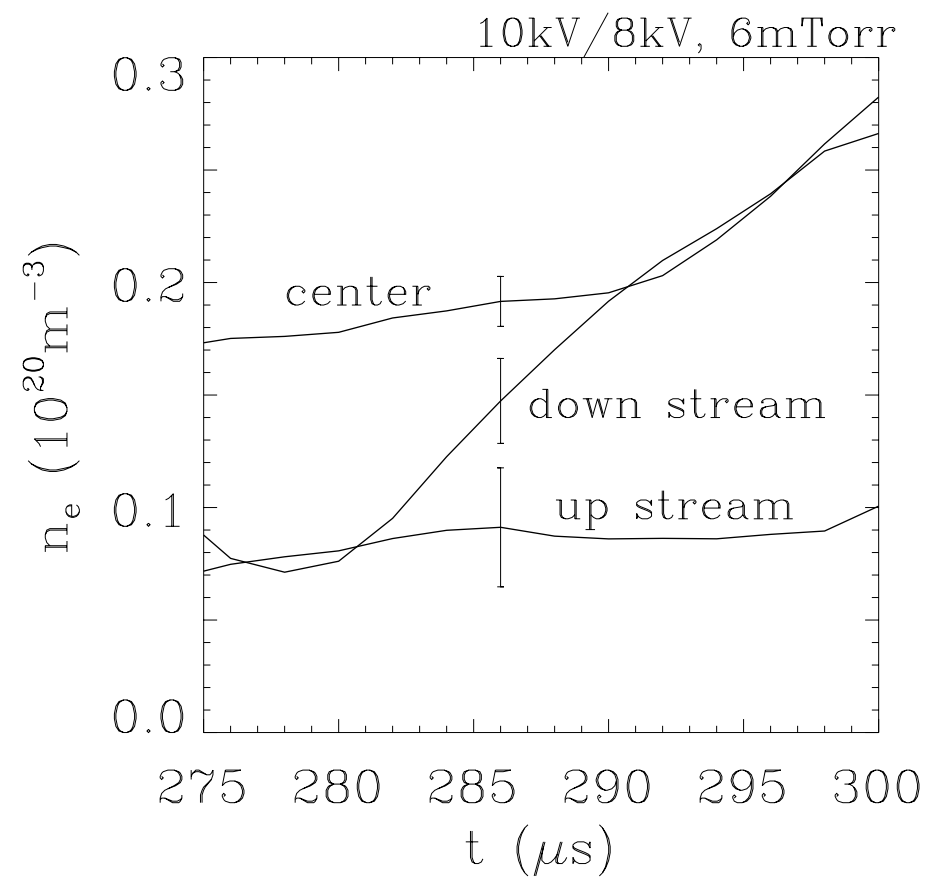

FIG. 7. Time evolution of density measured at center $(Z=0, R=37.5 \mathrm{~cm})$, up-stream $(Z=0, R=30 \mathrm{~cm}$ and $44 \mathrm{~cm})$, and down-stream $(Z=-10 \mathrm{~cm}, R=37.5 \mathrm{~cm})$ regions.

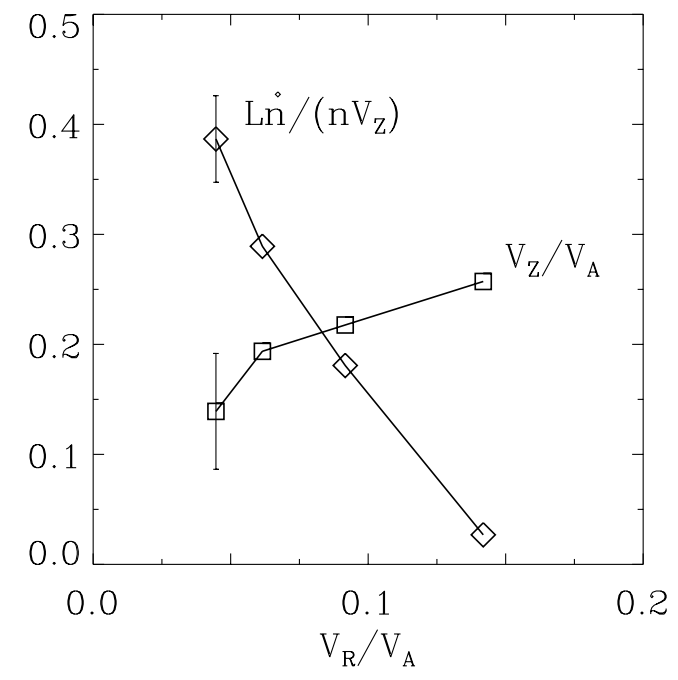

FIG. 8. Effects of compressibility (open diamonds) and downstream pressure (open squares) as functions of reconnection rate. As reconnection rates decreases, the outflow is further slowed by downstream pressure while the effect of compressibility becomes increasingly important. 


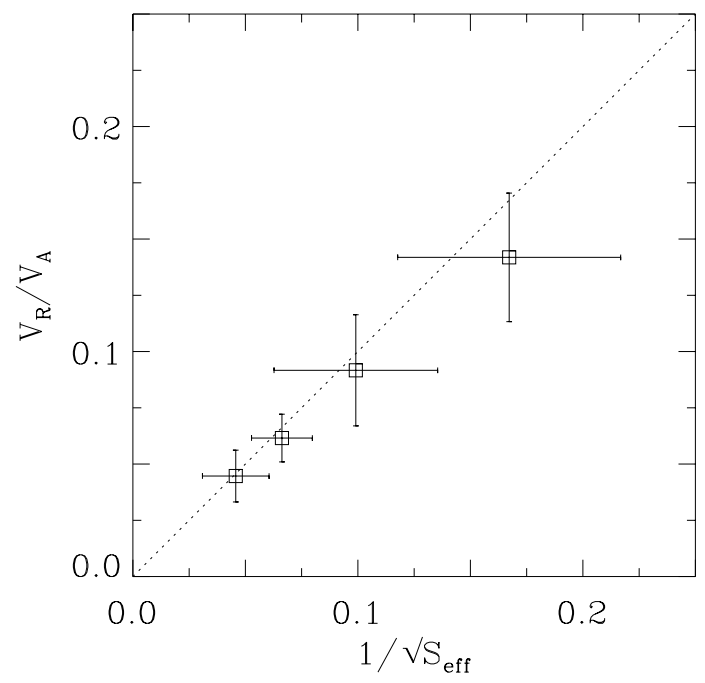

FIG. 9. The observed reconnection rates are compared to the prediction by a generalized Sweet-Parker model, $1 / \sqrt{S_{\text {eff }}}$, which incorporates finite compressibility, downstream pressure and the effective resistivity.

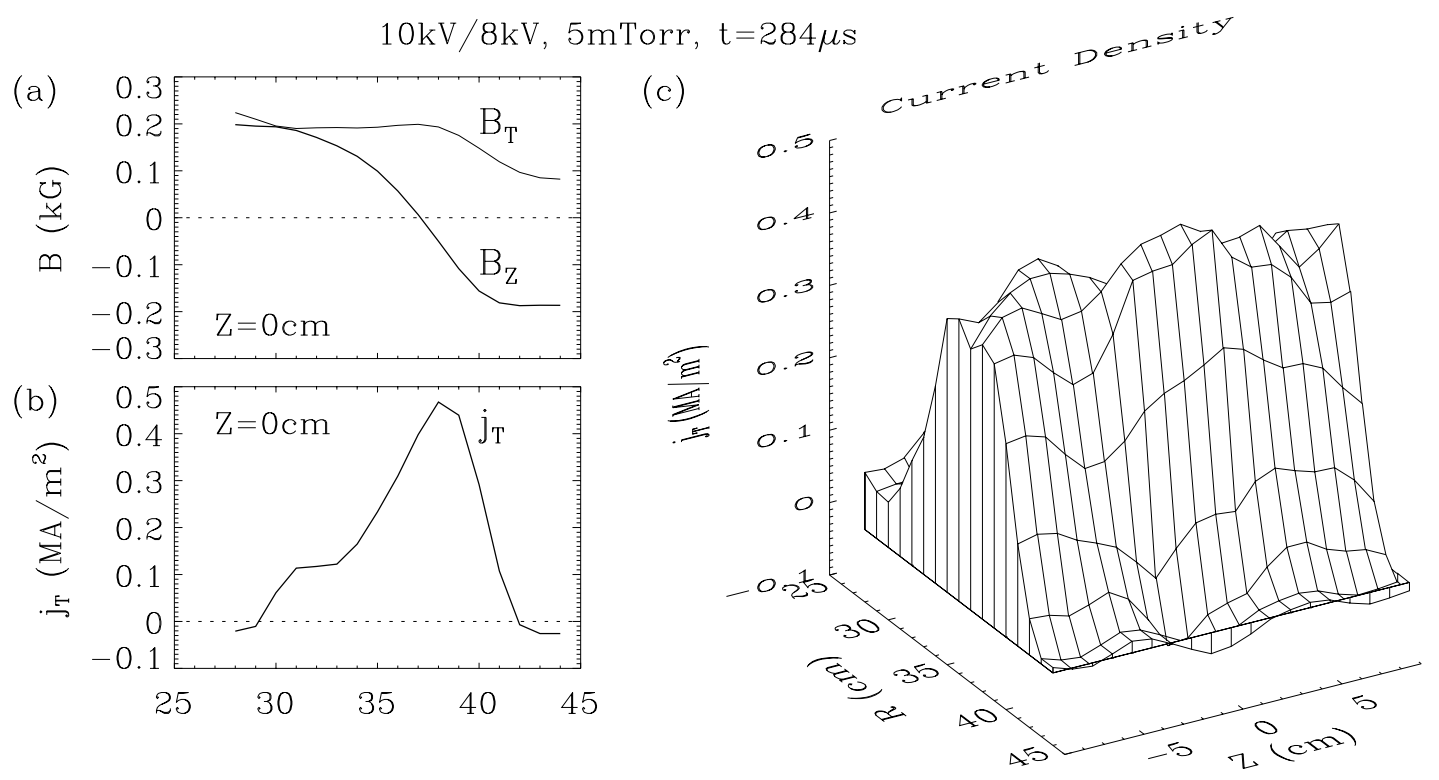

FIG. 10. The measured profile in the co-helicity case: (a) radial profiles of $B_{T}$ and $B_{Z}$ at $Z=0$, (b) radial profile of $j_{T}$ at $Z=0$, and (c) $j_{T}$ profile in $R-Z$ plane. 


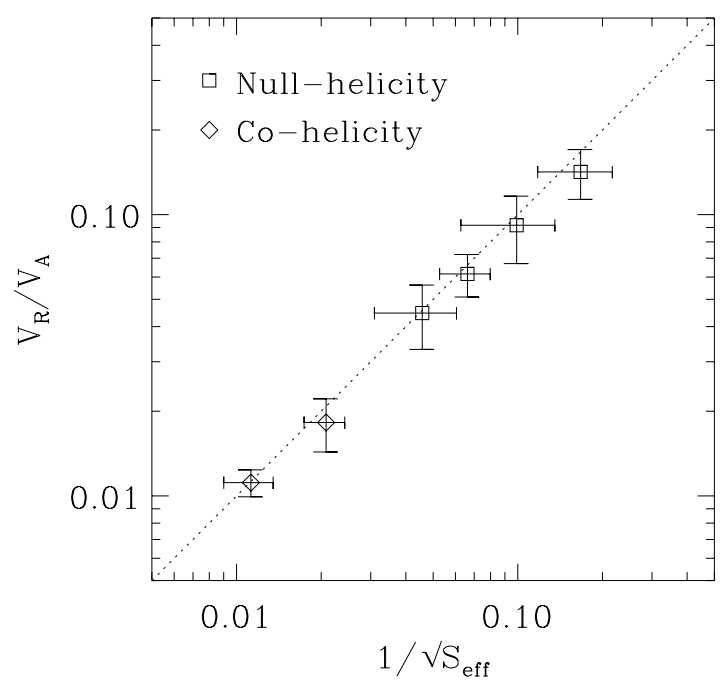

FIG. 11. The observed reconnection rates are compared to predictions by a generalized Sweet-Parker model for both null-helicity and co-helicity cases.
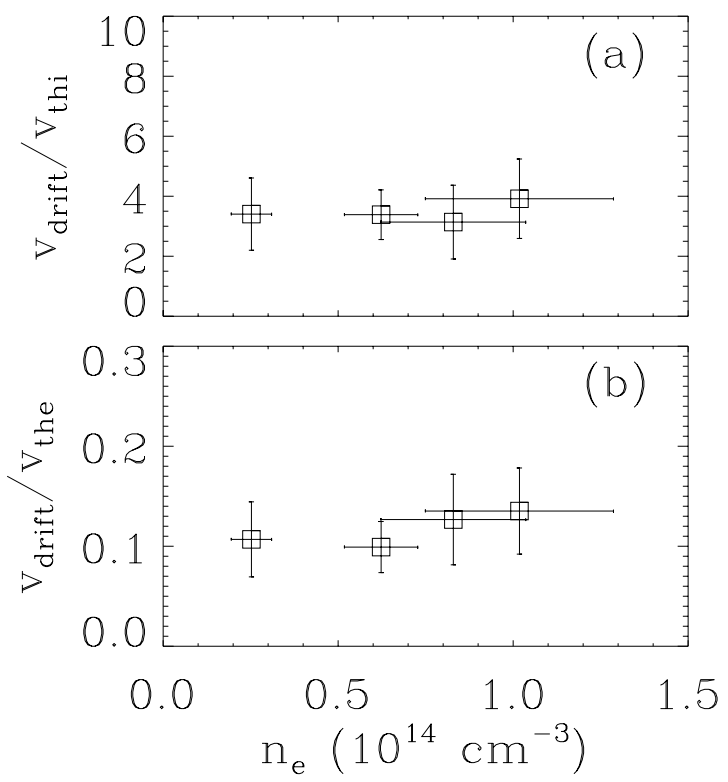

FIG. 12. The drift velocity when normalized by the ion thermal velocity (a) or the electron thermal velocity (b) as functions of density in the null-helicity case. 